ISASE 2019

\title{
A Classification Method for Silhouettes of Various Clothes
}

\author{
Tetsuo TSURU *, Masahiro SUGAHARA ** and Haruhiko NISHIMURA *** \\ * Ueda College of Fashion, 2-5-8 Shibata, Kita-ku, Osaka-shi, Osaka 530-0012, Japan \\ tsuru@uedagakuen.ac.jp \\ ** Proto Vision \\ protovision@yahoo.co.jp \\ *** University of Hyogo, 7-1-28 Minatojima-minami, Chuo-ku, Kobe, Hyogo 650-0047, Japan \\ haru@ai.u-hyogo.ac.jp
}

\begin{abstract}
In this paper, we propose an effective classification method for the silhouettes of various kinds of clothes. There are two approaches to analyzing clothes. The first method focuses on the basic elements of a garment's design, from the viewpoint of its creator. The second method features detailed categories with respect to the garment made. The silhouette of a garment is one of the most important pieces of information in fashion design trends. Here, we focused on classifying silhouettes that lead to the creation of trends, rather than classifying items made of clothing. This classification makes it possible to create a data set of silhouettes that can be used to multi-class classifications in a deep neural network.
\end{abstract}

Keywords: Silhouette, Classification, Fashion design, Multivariate analysis

\section{INTRODUCTION}

The use of deep neural networks and computer vision in the field of fashion field has recently increased significantly; it has been applied to coordinating the style of clothes, for example [1-7]. However, this research has focused only on the analytical evaluation of clothing items (e.g. jackets and skirts) and combinations thereof but has not been applied to the analytical evaluation of silhouettes and outlines. In fashion design, identifying the trend of an outfit is more important than its functionality. When creating a new design, visually understanding how a silhouette has changed from the past to the present is also important in predicting future designs.

In general, there are three basic elements of design: shape, color, and material. In fashion design, however, new trends are created for each season, and the "elements of shape" are divided into four elements: silhouette (outline), color, fabric, and detail. In the field of fashion design, trends change quickly, changing every season, such as spring/summer, midsummer, and autumn/winter. In order to respond to the speed of these trend changes,

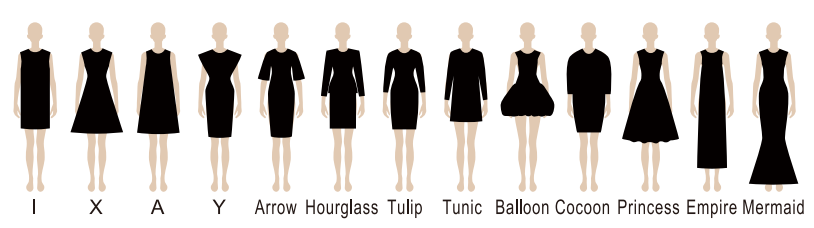

Figure 1: General silhouette classifications (I-line, X-line, A-line, etc.) fashion design decomposes the "element of shape" into silhouette and detail design.

A specific silhouette of a piece of clothing is often called a "*-line (e.g. X-line, I-line, Y-line, etc.)" in women's clothing, especially dresses and suits (two piece), and similar types of clothes. "Line" is an abbreviation of "silhouette line" and refers to the outline of the clothing's shape [8].

We have collected about 10,000 styling pictures, and have classified silhouettes by hand. As a result, many silhouettes have not been included in these categories, such as the silhouette classification (Fig. 1) [9] used in the apparel industry.

Therefore, in this study, we can create a data set for deep neural networks and computer vision to examine the classification method for silhouettes, for the purpose of clarifying the silhouette styles of various kinds of clothes.

\section{EXPERIMENTAL METHODS}

In this experiment, we implemented two approaches, a subjective viewpoint and an objective viewpoint. The subjective viewpoint was based on a biaxial silhouette, whereas the objective approach involved measuring the silhouette of the target image and quantifying it. We used three kinds of analytical methods, principal component analysis (PCA), a multidimensional scale (MDS) matrix, and cluster analysis, which are considered to contribute to 
silhouette classification from multivariate analysis [10].

[Analysis 1] PCA: This summarizes numerous quantitative explanatory variables into synthetic variables, as a basis for organizing the items of the silhouette classification. In this way, it classifies the relative contributions of each variable. Here, we aim to use PCA to determine which kind of classifications should be focused on.

[Analysis 2] MDS matrix: This visualizes the similarity of each silhouette as a point in multidimensional space, so that the distance and positional relationship of similarities between each silhouette becomes clear. It is useful for examining classification items. Here, we use a MDS matrix to classify the problem using a comparison between the subjective distribution map and the numerical distribution map. This ensures the reliability of the numerical distribution, allowing it to be used to discriminate the data set classification items of the deep layer neural net.

[Analysis 3] Cluster Analysis: The relationship of each silhouette can be clarified from a dendrogram obtained by Euclidean distance calculations, made following Ward's method[11]. Cluster analysis is useful for determining how to group the classification items.

In a previous study on silhouettes [12], the possibility of classification by feature was indicated by INUI et al. There, ten horizontal lines were set at equal intervals, from the starting point at the shoulder of the garment to the hem of the garment. Two points (the shoulder tip and the most constricted position) were added to ten points that intersected with the contours. The features of the silhouette were extracted from a total of 12 measurement positions. In this study, however, we consider that it is not possible to correspond the design points of various different silhouettes to equal intervals of measurement. We therefore measured eleven points at the measurement positions indicated in Fig. 2 when the pattern maker moved from the design picture to the pattern making. The styling images of this study were selected from the 201213 AW collections of luxury brands that have been presented in the Paris collection every season, downloaded from the FashionPRESS site [13].

Considering that the left and right sides of the silhouette may appear asymmetric depending on the way the fashion model walks, we considered it as the measurement position when creating the clothing pattern.

For measurement purposes, the image was resized so that the height of the image in question fell within a range of 122-130 mm, and the results of the measurements were analyzed using multivariate analysis, performed using the

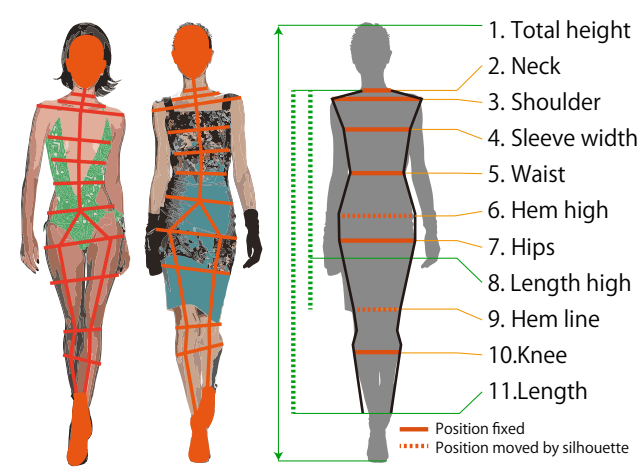

Figure 2: Measurement positions used in this study

Excel statistics package [10]. The measured sites were at the following 11 locations:

1. Total height

2. Neck

3. Shoulder

4. Sleeve width

5. Waist

6. Hem high (e.g. jackets hem widths)

7. Hips

8. Length high (Length high from Neck)

9. Hem line (width)

10.Knee

11.Length (Length from Neck)

\section{RESULTS AND DISCUSSION}

\subsection{Analysis 1: PCA}

PCA 1 is $45.10 \%$, PCA 2 is $20.95 \%$, and PCA 3 is $14.21 \%$; and the cumulative contribution ratio of PCA 1 to 3 is $80.26 \%$. In the upper values of the main component loading amounts (Fig. 3), the main components of PCA lare Knee 0.9508 , Hips 0.9309 , Hem high 0.8315 , and Hem Line 0.7916. whereas the waist is a more minor component. PCA 2 has s shoulder value of 0.7786 , however the waist value, 0.7529 , is much higher than in 1 . PCA 3 has a Neck value of 0.9609 , it is considered to be the axis of the identity of the silhouette, because the fluctuation widths of the other parts are small.

In PCA 1 vs. PCA 2 (Fig. 4), there is a neck-point at the center and other points such as shoulder, waist, hem high, hips, knee, hem line are positioned to surround the periphery. Therefore, the neck-point is considered to be the starting point when the feature quantity of the whole silhouette is expressed.

\subsection{Analysis 2: MSD}

In the distribution map of MDS (Fig. 5, left figure), groups are divided for each feature quantity. Group A is a long dress of A-line type, with a hem line going down to 
the feet. Group B is an I-line type garment with a wholly slim silhouette, and group C is an X-line type silhouette, whose waist is narrowed. Although the number of silhouettes of clothes that do not belong to the group is small, there is a possibility of an important silhouette for examining a new group name. The interpretation of silhouettes not belonging to the $\mathrm{A}, \mathrm{B}$, or $\mathrm{C}$ groups is divided into two. First, interpret it as one as another. The second is interpretation is divided into EFG and small groups are considered as a new group. In this study, we considered the second interpretation. This is because we think that there is a possibility of finding a new silhouette in silhouettes not belonging to the latter group to realize silhouette classification for hundreds of thousands of images. In the placement diagram of this subjective silhouette (Fig. 5, right figure), manual placement was performed by plotting "thin/thick" on the horizontal axis and "voluminous tops in high positions/voluminous bottoms in low positions" on the vertical axis.
We compared the positions of the same garments on the left and right silhouette distribution maps in Fig. 4. A line was drawn from the silhouette distribution chart on the right figure to the left figure. The figure on the right is a subjective impression, in which clothing is classified into three types: X-line (green line), A-line (red line), and Iline (blue line). As a result of this analysis, the existence of clothing types other than those in groups A, B, and C on the left figure could be seen, and their features became clear.

\subsection{Analysis 3: Cluster analysis}

A silhouette dendrogram was created from the cluster analysis (Fig. 6). The broken line in the figure is divided into four clusters, and the relationships of the silhouettes are arranged in a tree structure. Comparing this dendrogram to $\mathrm{ABC}$ groups from the MDS method (Fig. 5) however, shows that cluster analysis has divided the $C$ group into two; there is a silhouette other than the $\mathrm{ABC}$ groups.

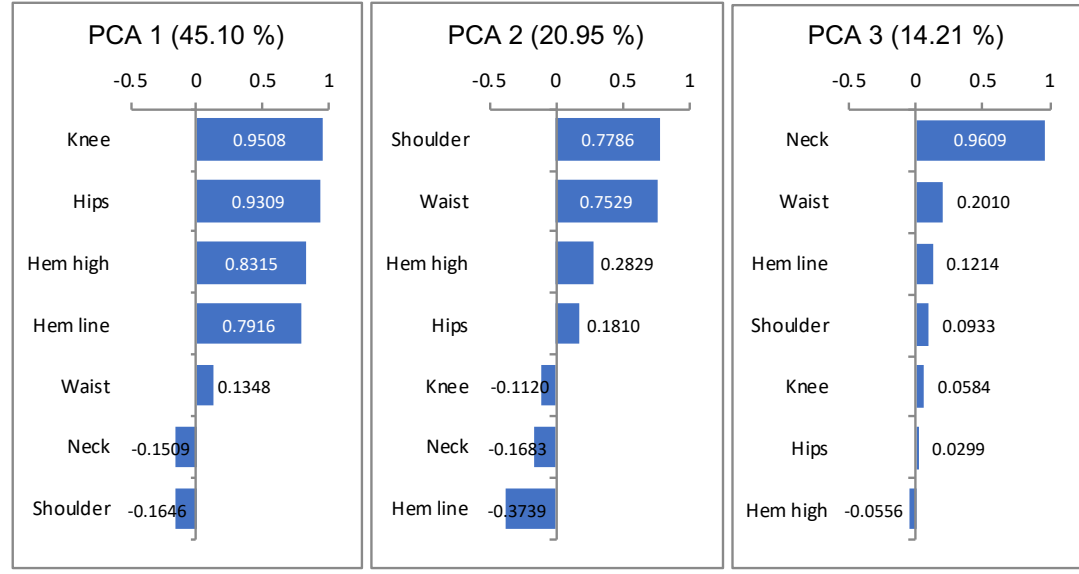

Figure 3: Principal component loading amounts

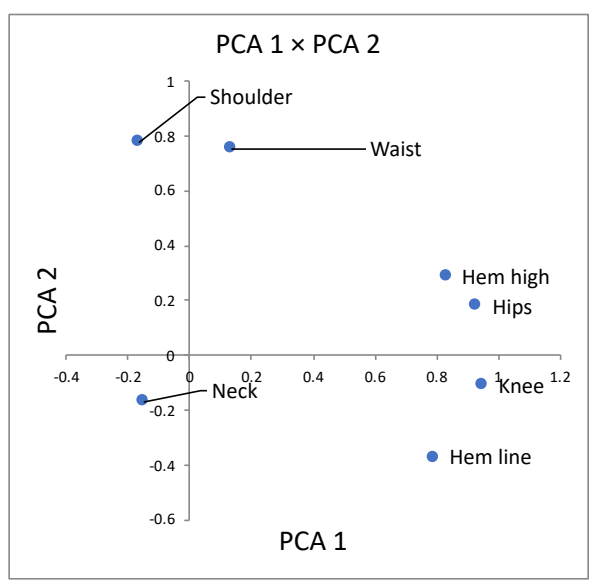

Figure 4: Relationship between PCA 1 and PCA 2

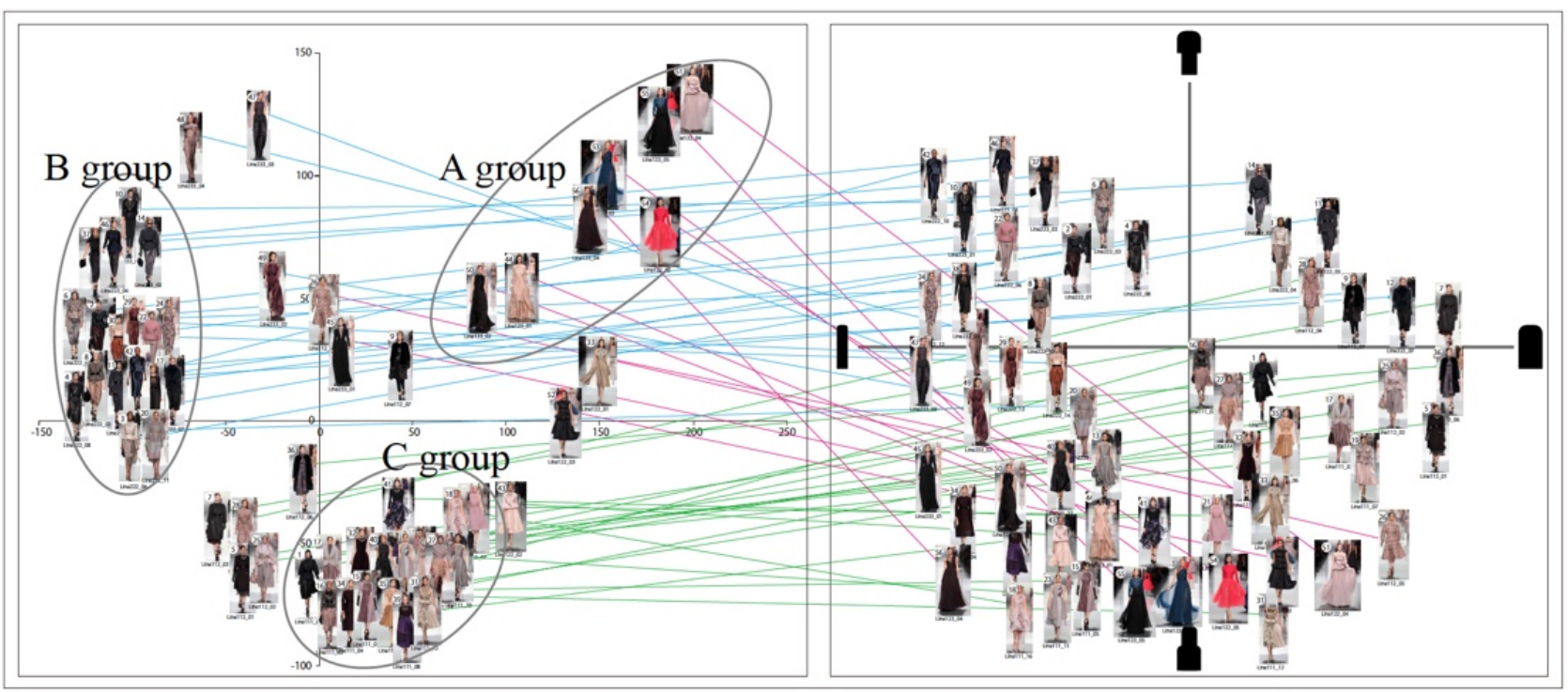

Figure 5: Silhouette distribution (left: MDS method, right: manual arrangement) 


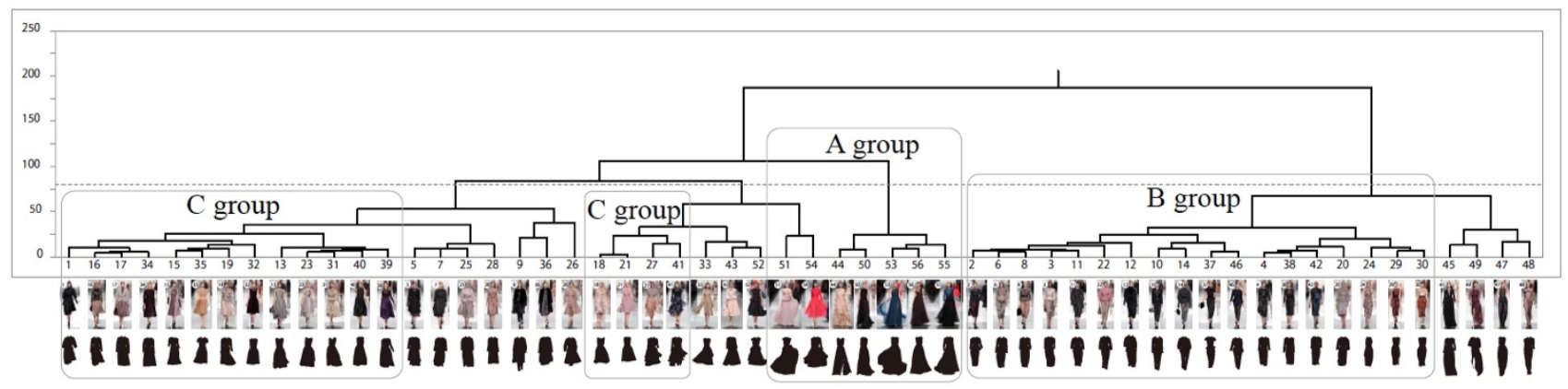

Figure 6: Dendrogram of silhouette classifications

\section{CONCLUSION}

Here, we have detailed a classification method for performing silhouette classifications for various kinds of clothes. As a result, we have shown that it is possible to create classifications corresponding to various silhouettes by implementing numerical analysis. However, it was also revealed that, in addition to groups identified by MDS analysis, and cluster analysis, a further group is necessary. Future research is needed to name and define this final group.

Past studies have used a data set of subjective classifications when classifying common silhouettes. This data set was prepared by using the general classification criteria of silhouettes. However, in the process of classification by hand, many doubts as to which silhouette group the selected styling belongs to occurred. For this reason, it was found that it is difficult to create a data set that can be used for classification of various silhouettes using the general silhouette classification standard based approach. In this study, we have showed that it is possible to create classifications corresponding to new feature quantities of silhouettes, and to create data sets that correspond to multiple classifications.

Future studies should focus on the appropriate naming of classified silhouette groups, the expansion of data sets for multiple classifications, and the construction of multiple classification networks using deep layer neural networks.

\section{REFFERENCES}

[1] Borras, Agnés, Francesc Tous, Josep Lladós and Maria Vanrel; High-level clothes description based on colour-texture and structural features, Pattern Recognition and Image Analysis, pp. 108-116, 2003.

[2] Bossard, Lukas, Matthias Dantone, Christian Leistner, Christian Wengert, Till Quack and Luc Van Gool., and Van Gool, L.; Apparel classification with style, in ACCV, pp. 321-335, Springer, 2012.
[3] Chen Hong, Zi Jian Xu, Zi Qiang Liu and Song Chun Zhu; Composite templates for cloth modeling and sketching, CVPR, Vol. 1, pp. 943- 950, 2006.

[4] Chen Huizhong, Andrew Gallagher and Bernd Girod; Describing clothing by semantic attributes, ECCV, pp. 609-623, 2012.

[5] Chen, Qiang, Junshi Huang, Rogerio Feris, Lisa M. Brown, Jian Dong, and Shuicheng Yan; Deep Domain Adaptation for Describing People Based on Fine-Grained Clothing Attributes, CVPR, pp. 53155324, 2015.

[6] Dong, Jian, Qiang Chen, Wei Xia, Zhongyang Huang, and Shuicheng Yan; A deformable mixture parsing model with parselets, ICCV, pp. 34083415, 2013.

[7] He Ruining, and Julian McAuley; Ups and Downs: Modeling the Visual Evolution of Fashion Trends with One-Class Collaborative Filtering, WWW, pp. 507-517, 2016.

[8] Fashion Business [I] revised edition, Japan Fashion Education Promotion Association, p188, 2008.

[9] Fashion Sales 3, Fashion Sales Capability Examination Grade 3 Grade Official Text, Japan Fashion Education Promotion Association, pp.102105, 2018.

[10] BellCurve for Excel (Multivariate analysis): https://bellcurve.jp/ex/

[11] Adachi, K. (Ed.) (2006). Tahenryol̀,, deÌ,,ta kaisekihoÌ,, [Multivariate data analysis method]. Kyoto, Japan: Nakanishiya Shuppan.

[12] Shigeru Inui, Keiko Miyatake, Yosuke Horiba; Study on Possibility of Silhouette Discrimination, https://kaken.nii.ac.jp/report/KAKENHI-PROJECT24220012/242200122015jisseki/\#product_36 (accessed on 1/29/2019).

[13] FashionPRESS: https://www.fashion-press.net/ (accessed on 11/11/2018). 